\title{
Brain imaging to be on track for improving diagnosis and pathophysiological insights in neuropsychiatric diseases
}

\author{
Andrea Schmitt • Peter Falkai
}

Published online: 29 August 2013

(C) Springer-Verlag Berlin Heidelberg 2013

Brain imaging techniques are becoming increasingly important in diagnosis and course of neuropsychiatric diseases. This is best demonstrated in dementia, where besides the volumetric investigation of the hippocampus, single-photon emission-computed tomography (SPECT) revealed hyperperfusion patterns in its several subtypes. International guidelines have suggested SPECT to be useful in differentiating several forms of dementia such as Alzheimer's disease, vascular dementia, frontotemporal dementia and dementia with Lewy bodies. In a metaanalysis and review, Yeo et al. [1] calculated sensitivity and specifity of ${ }^{99 \mathrm{~m}}$ Tc-HMPAO-SPECT in distinguishing dementia subtypes. They found SPECT investigations of diagnostic value, particularly in differentiating Alzheimer's dementia from frontotemporal dementia and healthy controls, adding information to the clinical test results. In addition, cerebrospinal fluid (CSF) markers are recommended for dementia diagnosis. Guo et al. [2] investigated protein expression in healthy controls, patients with mild cognitive impairment (MCI) and Alzheimer's dementia and found heart-type fatty acid-binding protein and vascular endothelial growth factor in CSF to distinguish between patients with Alzheimer's dementia and healthy controls with lower sensitivity and higher specificity and to predict the progression of MCI to dementia. They recommend combining these markers with the three established markers Amyloid-\$1-42, total-Tau and phosphorylatedTau, increasing sensitivity to $83 \%$ and specifity to $86 \%$.

A. Schmitt $(\square)$. P. Falkai

Department of Psychiatry and Psychotherapy, Ludwig-

Maximilians-University Munich, Nußbaumstr. 7, 80336 Munich, Germany

e-mail: Andrea.Schmitt@med.uni-muenchen.de
Adults with attention-deficit hyperactivity disorder (ADHD) consistently have been reported to exhibit working memory deficits, contributing to inattentive behavior and hyperactivity. Using a functional magnetic resonance imaging (fMRI) paradigm, Ko et al. [3] demonstrated that during a two-back working memory task, indeed both the adults with ADHD and healthy controls activate the frontoparietal network but ADHD patients to reveal higher brain activity over the bilateral anterior cingulate cortex, left inferior frontal lobe, hippocampus and supplementary motor area. Increasing the task loading to 3-back task, the control group showed increased brain activation, while the ADHD group exhibited greater decrease in brain activation over the left fronto-parietal network, suggesting impaired fronto-parietal network when demands of working memory exceed the capacity of patients with ADHD.

Post-traumatic stress disorder (PTSD) includes key symptoms as anxiety, hyperarousal, dissociative disturbances and a restricted range of emotions, called emotional numbing. To identify neuronal circuitry involved in emotional dysfunction, Mazza et al. [4] via fMRI investigated subjects with PTSD while presenting pictures with negative emotional valence versus neutral stimuli. Compared to healthy controls, PTSD patients showed hyperactivation in the left posterior insula, exerting influence on subcortical brain regions. These alterations may underly the emotional, social and relational difficulties in PTSD. Being an important interface between emotion and cognition, the anterior cingulate cortex (ACC) is strongly connected to the amygdala and involved in fear response regulation. In a structural MRI study, Jatzko et al. [5] delineated the ACC in 15 PTSD victims. While detecting no differences in absolute ACC volumes between patients and controls, they found one pattern, namely the single segmented cingulate sulcus, to be predominant in PTSD subjects. Further 
investigations in larger samples should assess to which extent alterations of ACC patterns may influence the incidence and symptoms of PTSD.

In antisocial personality, often being involved in aggression and violence, brain structural abnormalities in regions involved in social cognition and emotion such as the orbitofrontal cortex, amygdala or fronto-temporo-parietal cortical areas have been reported. In a structural MRI study, Bertsch et al. [6] were the first to compare male antisocial offenders with borderline personality (ASPDBPD) disorder but low psychopathic traits to subjects with antisocial personality and high psychopathic traits (ASPDPP) and healthy controls. They found specific alterations in ASPD-BPD in orbitofrontal and ventromedial prefrontal cortices subserving emotion regulation and reactive aggression. The group specifically detected decreased volumes of the dorsomedial prefrontal and posterior cingulate cortex as well as postcentral gyrus in ASPD-PP, which probably reflects neural correlates of callousness and poor moral judgment. However, results in different criminal offender groups should be replicated in larger samples. Schiltz et al. [7] investigated MRI and CT scans from 287 male prison inmates not diagnosed as mentally ill compared to 52 non-criminal controls. In this study, violent offenders displayed a higher rate or morphological abnormalities in the frontal and parietal cortex, ventricles and medial temporal structures compared to non-violent offenders and controls. These brain structures are involved in the control of social and violent behavior, for which reason brain imaging may be a diagnostic tool to detect presence of structural brain damage in violent offenders. Overall, MRI investigations are on the way to provide insights in disturbed neuronal circuitry in neuropsychiatric conditions, trauma-related diseases and even personality disorders, leading to improved diagnostic approaches in the future.

\section{References}

1. Yeo JM, Lim X, Khan Z, Pal S (2013) Systematic review of the diagnostic utility of SPECT imaging in dementia. Eur Arch Psychiatry Clin Neurosci. doi:10.1007/s00406-013-0426-z

2. Guo L-H, Alexopoulos P, Perneczky R (2013) Heart-type fatty acid binding protein and vascular endothelial growth factor: cerebrospinal fluid biomarker candidates for Alzheimer's disease. Eur Arch Psychiatry Clin Neurosci. doi:10.1007/s00406-0130405-4

3. Ko C-H, Yen J-Y, Yen C-F, Chen C-S, Lin W-C, Wang P-W, Liu G-C (2013) Brain activation deficits in increased-load working memory tasks among adults with ADHD using fMRI. Eur Arch Psychiatry Clin Neurosci. doi:10.1007/s00406-013-0407-2

4. Mazza M, Tempesta D, Pino MC, Catalucci A, Gallucci M, Ferrara M (2013) Regional cerebral changes and functional connectivity during the observation of negative emotional stimuli in subjects with post-traumatic stress disorder. Eur Arch Psychiatry Clin Neurosci. doi:10.1007/s00406-013-0394-3

5. Jatzko A, Vogler C, Demirakca T, Ruf M, Malchow B, Falkai P, Braus DF, Ende G, Schmitt A (2013) Pattern and volume of the anterior cingulate cortex in chronic posttraumatic stress disorder (PTSD). Eur Arch Psychiatry Clin Neurosci. doi:10.1007/s00406013-0408-1

6. Bertsch K, Grothe M, Prehn K, Vohs K, Berger C, Hauenstein K, Keiper P, Domes G, Teipel S, Herpertz SC (2013) Brain volumes differ between diagnostic groups of violent criminal offenders. Eur Arch Psychiatry Clin Neurosci. doi:10.1007/s00406-013-0391-6

7. Schiltz K, Witzel JG, Bausch-Hölterhoff J, Bogerts B (2013) High prevalence of brain pathology in violent prisoners: a qualitative $\mathrm{CT}$ and MRI scan study. Eur Arch Psychiatry Clin Neurosci. doi:10. 1007/s00406-013-0403-6 